\title{
Neutrino Mass and Baryon Asymmetry from Dirac Seesaw
}

\author{
Pei-Hong $\mathrm{Gu}^{1,27}$ and Hong-Jian He倜 \\ ${ }^{1}$ Center for High Energy Physics, Tsinghua University, Beijing 100084, China \\ ${ }^{2}$ The Abdus Salam International Centre for Theoretical Physics, Strada Costiera 11, I-34014 Trieste, Italy
}

\begin{abstract}
We extend the visible content of the standard model (SM) with a hidden sector composed of three right-handed singlet neutrinos and one singlet Higgs. These extra singlets are charged under a new $U(1)_{X}$ gauge symmetry while the SM particles are not. Two heavy scalar doublets are introduced to play the role of the messengers between the visible and hidden sectors. The neutrinos naturally acquire tiny Dirac masses because the ratio of weak scale over the heavy messenger masses is highly suppressed. Furthermore, the heavy messengers simultaneously generate baryon asymmetry of the universe through their out-of-equilibrium CP-violating decays.
\end{abstract}

PACS: 98.80.-k; 11.30.Er, 14.60.Pq

$[$ hep-ph/0610275]

The tiny neutrino masses and the matter-antimatter asymmetry in the universe pose two major challenges to particle physics and cosmology. This indicates the necessity of supplementing to the existing theory certain new ingredients, which have been hidden from the direct experimental observations so far [1].

In this paper, we propose a novel Dirac Seesaw model to obtain the tiny neutrino masses and generate the observed baryon asymmetry by extending the visible content of the standard model (SM) with a hidden sector which is prescribed by a $U(1)_{X}$ gauge symmetry and composed of three right-handed singlet neutrinos plus one singlet Higgs. Two heavy scalar doublets are introduced to play the role of messengers between the visible and hidden sectors.

The field content of the proposed $S U(2)_{L} \otimes U(1)_{Y} \otimes$ $U(1)_{X}$ model is defined in Table【 where $\psi_{L}, \phi, \nu_{R}, \chi$, $\eta$ are the left-handed lepton doublets, the Higgs doublet, the right-handed singlet neutrinos, the singlet Higgs and the heavy scalar doublets, respectively. All other unlisted SM fields are $U(1)_{X}$ singlets. In this Table we have also suppressed the generation indices for simplicity. It is clear that the conventional Yukawa couplings of the left-handed lepton doublets and the right-handed singlet neutrinos with the light Higgs doublet $\phi$ are forbidden because the right-handed neutrinos have $U(1)_{X}$ charge while all the SM particles do not. Namely, the sector composed of the right-handed neutrinos and the Higgs singlet is hidden from the visible SM sector. However, the heavy scalar doublets $\eta$, which join not only the SM gauge group $S U(2)_{L} \otimes U(1)_{Y}$ but also the new $U(1)_{X}$, can bridge the two sectors. In this sense, we may regard the heavy scalar doublets as "messengers".

We can thus write down the relevant interaction Lagrangian for generating the Dirac neutrino masses. It involves the messenger scalars $\eta$, the SM-like doublet $\phi$ and the singlet scalar $\chi$,

$$
\mathcal{L} \supset-y \bar{\psi}_{L} \eta \nu_{R}+\rho \chi \eta^{\dagger} \phi+\text { h.c. },
$$

*Electronic address: pgu@ictp.it

${ }^{\dagger}$ Electronic address: hjhe@tsinghua.edu.cn

\begin{tabular}{c|ccc}
\hline \hline Fields & $S U(2)_{L}$ & $U(1)_{Y}$ & $U(1)_{X}$ \\
\hline$\psi_{L}$ & $\mathbf{2}$ & $-\frac{1}{2}$ & 0 \\
$\phi$ & $\mathbf{2}$ & $-\frac{1}{2}$ & 0 \\
$\nu_{R}$ & $\mathbf{1}$ & 0 & $-\frac{1}{2}$ \\
$\chi$ & $\mathbf{1}$ & 0 & $+\frac{1}{2}$ \\
$\eta$ & $\mathbf{2}$ & $-\frac{1}{2}$ & $+\frac{1}{2}$ \\
\hline \hline
\end{tabular}

TABLE I: The field content related to the generation of the Dirac neutrino masses. All other SM fields are $U(1)_{X}$ singlets and the family indices have been omitted for simplicity.

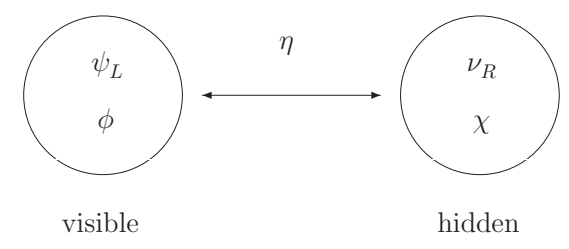

FIG. 1: The heavy scalar doublets $\eta$ act as the messengers between the visible and hidden sectors. The other SM fields and the family indices have been omitted for simplicity.

where $y$ is the Yukawa coupling. The cubic scalar coupling $\rho$ has mass dimension- 1 and is set to be real after a proper phase rotation. From Fig.2, we note that at low energy we can integrate out the heavy messengers $\eta$ and obtain the following effective dimension- 5 operator,

$$
\mathcal{L}_{\text {Dirac }}^{(5)}=-y \frac{\rho}{M_{\eta}^{2}} \bar{\psi}_{L} \phi \chi \nu_{R}+\text { h.c. },
$$

where $M_{\eta}$ denotes the heavy messenger masses. So, once the Higgs fields $\phi$ and $\chi$ get nonzero vacuum expectation values (VEVs) after the symmetry breaking, the neutri- 


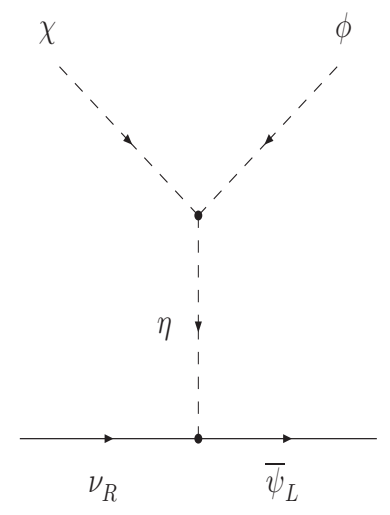

FIG. 2: The generation of Dirac neutrino masses. Here the family indices have been suppressed.

nos naturally acquire small Dirac masses,

$$
M_{\nu} \simeq y \frac{\rho\langle\phi\rangle\langle\chi\rangle}{M_{\eta}^{2}}=y \frac{\rho v u}{M_{\eta}^{2}} .
$$

Here $\langle\phi\rangle \equiv v$ and $\langle\chi\rangle \equiv u$ represent the scalar VEVs. From minimizing the scalar potential in Appendix-A, we have also shown that the heavy messengers will acquire tiny VEVs, $\langle\eta\rangle \simeq \rho u v / M_{\eta}^{2}$, after $S U(2)_{L} \otimes U(1)_{Y} \otimes$ $U(1)_{X}$ is broken to $U(1)_{\mathrm{em}}$. This confirms Eq. (3) due to the mass formula $M_{\nu}=y\langle\eta\rangle$ from Eq. (11).

The vacuum expectation value $\langle\chi\rangle=u$ breaks $U(1)_{X}$ and is expected to be nearby the weak scale, so we will set $u=\mathcal{O}(\mathrm{TeV})$. As for the cubic scalar coupling $\rho$, we consider a reasonable case where $\rho$ is of order $M_{\eta}$ or less. Under this setup, it is easy to see from Eq. (3) that the Dirac neutrino masses will be efficiently suppressed by the ratio of the weak scale over the heavy messenger masses. For instance, we find that, for $M_{\eta} \sim 10^{14} \mathrm{GeV}$, $\rho \sim 10^{13} \mathrm{GeV}$ and $y \sim \mathcal{O}(1)$, the neutrino masses can be naturally around $\mathcal{O}(0.1 \mathrm{eV})$. It is clear that this new mechanism of the neutrino mass generation has two essential features: (i) it generates Dirac masses for neutrinos, and (ii) it retains the essence of the conventional seesaw [2] by making the neutrino masses tiny via the small ratio of the weak scale over the heavy mass scale, and hence may be called Dirac Seesaw. We further note that the above Dirac-type dimension-5 effective operator (2) does share certain essential features with the traditional Majorana-type dimension-5 effective operator [3],

$$
\mathcal{L}_{\text {Majorana }}^{(5)}=-\frac{c}{\Lambda} \psi_{L}^{T} \epsilon \widetilde{\phi} \mathcal{C} \widetilde{\phi}^{T} \epsilon \psi_{L}+\text { h.c. },
$$

where $\widetilde{\phi}=i \tau_{2} \phi^{*}$ has hypercharge $+\frac{1}{2}$ and $\mathcal{C}=i \gamma^{2} \gamma^{0}$ is the charge-conjugation operator. The major difference of the current Dirac Seesaw from the conventional Majorana Seesaw 2] is that the former involves heavy doublet scalar rather than heavy singlet Majorana fermion. After integrating out the heavy fields (either the heavy messenger scalars in the Dirac Seesaw or the heavy right-handed neutrinos in the Majorana Seesaw), we see that the resulted effective dimension-5 operators (2) and (4) share an essential similarity regarding the generation of small neutrino masses.

In the following, we demonstrate how to generate the observed baryon asymmetry within the above Dirac Seesaw scenario. It allows us to make use of the so-called Dirac leptogenesis mechanism [4]. As shown in [4], since the sphalerons [5] have no direct effect on the righthanded fields, a nonzero lepton asymmetry stored in the right-handed fields could survive above the electroweak phase transition and then produce the baryon asymmetry in the universe, although the lepton asymmetry stored in the left-handed fields had been destroyed by the sphalerons. For all the SM species, the Yukawa couplings are sufficiently strong to rapidly cancel the stored left- and right-handed lepton numbers. But the effective interactions of the right-handed Dirac neutrinos are exceedingly weak, and the equilibrium between left- and right-handed lepton numbers will not be realized until temperatures fall well below the weak scale. At that time the left-handed lepton number has already been converted to baryon number by the sphalerons. Hence, the leptogenesis [6] could be valid even if the neutrinos are not of Majorana nature [7]. For the SM with three generation fermions and one Higgs doublet, one finds [4] that the final baryon number, which is converted from the lepton number stored in the left-handed leptons, should be

$$
n_{B}=n_{L}=-\frac{28}{79} n_{\nu_{R}} .
$$

In the Dirac leptogenesis scenario, the requirement of $\mathrm{CP}$ violation must be fulfilled in order to generate a baryon asymmetry in the early universe. In the present model, the heavy messengers have two decay processes:

$$
\eta \rightarrow\left\{\begin{array}{c}
\psi_{L} \nu_{R}^{c} \\
\phi \chi
\end{array}, \quad \eta^{\dagger} \rightarrow\left\{\begin{array}{c}
\psi_{L}^{c} \nu_{R} \\
\phi^{\dagger} \chi^{\dagger}
\end{array}\right.\right.
$$

The channels of $\eta \rightarrow \psi_{L} \nu_{R}^{c}$ and $\eta^{\dagger} \rightarrow \psi_{L}^{c} \nu_{R}$ could provide the expected asymmetry between the right-handed neutrinos and antineutrinos as long as the CP is not conserved. For the purpose to produce CP-violation, we need at least two such heavy messenger scalars $\eta_{j}(j=1,2)$ to realize the interference between the tree diagram and the irreducible loop correction. Here we minimally introduce two heavy messengers and write down the relevant Lagrangian,

$\mathcal{L} \supset \sum_{j=1}^{2}\left[-M_{\eta_{j}}^{2} \eta_{j}^{\dagger} \eta_{j}-y_{j \alpha \beta} \bar{\psi}_{L \alpha} \eta_{j} \nu_{R \beta}+\rho_{j} \chi \eta_{j}^{\dagger} \phi+\right.$ h.c. $]$.

where the summation over the family indices $(\alpha, \beta)$ is implied. Fig. 3 depicts the decay of $\eta_{i} \rightarrow \psi_{L \alpha} \nu_{R \beta}^{c}$ at tree-level and one-loop order. Then, we can define the 


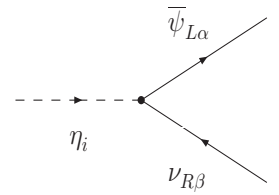

(a)

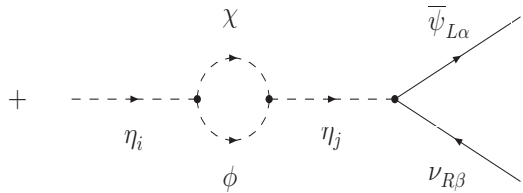

(b)
FIG. 3: The decay of $\eta_{i} \rightarrow \psi_{L \alpha} \nu_{R \beta}^{c}$ at tree-level (a), and at one-loop order (b). The interference of these diagrams produces the CP-violation needed for giving rise to a net asymmetry stored in the right-handed neutrinos.

CP asymmetry for the right-handed neutrinos,

$\varepsilon_{R i} \equiv \frac{\sum_{\alpha \beta}\left[\Gamma\left(\eta_{i}^{\dagger} \rightarrow \psi_{L \alpha}^{c} \nu_{R \beta}\right)-\Gamma\left(\eta_{i} \rightarrow \psi_{L \alpha} \nu_{R \beta}^{c}\right)\right]}{\Gamma_{i}}$,

where $\Gamma\left(\eta_{i} \rightarrow \psi_{L \alpha} \nu_{R \beta}^{c}\right)$ is the decay width of $\eta_{i} \rightarrow$ $\psi_{L \alpha} \nu_{R \beta}^{c}$, and so on. $\Gamma_{i}$ denotes the total decay width of $\eta_{i}$ or $\eta_{i}^{\dagger}$

$$
\begin{aligned}
\Gamma_{i} & \equiv \sum_{\alpha \beta} \Gamma\left(\eta_{i} \rightarrow \psi_{L \alpha} \nu_{R \beta}^{c}\right)+\Gamma\left(\eta_{i} \rightarrow \phi \chi\right) \\
& \equiv \sum_{\alpha \beta} \Gamma\left(\eta_{i}^{\dagger} \rightarrow \psi_{L \alpha}^{c} \nu_{R \beta}\right)+\Gamma\left(\eta_{i}^{\dagger} \rightarrow \phi^{\dagger} \chi^{\dagger}\right) .
\end{aligned}
$$

Note that the second equality in (9) is guaranteed by the unitarity and the CPT conservation. In comparison with the right-handed neutrinos, the left-handed leptons should have an equal but opposite CP asymmetry. After a lengthy calculation, we derive the total decay width and the CP asymmetry, respectively,

$$
\begin{gathered}
\Gamma_{i}=\frac{1}{8 \pi}\left[\operatorname{Tr}\left(y_{i}^{\dagger} y_{i}\right)+\frac{\rho_{i}^{2}}{M_{\eta_{i}}^{2}}\right] M_{\eta_{i}}, \\
\varepsilon_{R i}=-\frac{1}{4 \pi} \frac{\operatorname{Im}\left[\operatorname{Tr}\left(y_{i}^{\dagger} y_{j}\right) \rho_{i} \rho_{j}\right]}{\operatorname{Tr}\left(y_{i}^{\dagger} y_{i}\right) M_{\eta_{i}}^{2}+\rho_{i}^{2}} \frac{M_{\eta_{i}}^{2}}{M_{\eta_{i}}^{2}-M_{\eta_{j}}^{2}},
\end{gathered}
$$

where $j \neq i$.

In the case that the two heavy messengers have hierarchical masses, the final lepton asymmetry comes mainly from the decay of the lighter one. For illustration, let us focus on this hierarchical case. Without losing generality, we will choose $\eta_{1}$ to be the ighter messenger and $\eta_{2}$ the heavier one. Thus, we can simplify the CP-asymmetry as,

$$
\varepsilon_{R 1} \simeq \frac{1}{4 \pi} \frac{\operatorname{Im}\left[\operatorname{Tr}\left(y_{1}^{\dagger} y_{2}\right)\right] \rho_{1} \rho_{2}}{\operatorname{Tr}\left(y_{1}^{\dagger} y_{1}\right) M_{\eta_{1}}^{2}+\rho_{1}^{2}} \frac{M_{\eta_{1}}^{2}}{M_{\eta_{2}}^{2}}
$$

where the cubic couplings $\rho_{1}$ and $\rho_{2}$ are properly defined to be real as mentioned below Eq. (11). The presence of the relative phases between $y_{1}$ and $y_{2}$ can generate a net lepton asymmetry stored in the right-handed neutrinos via the out-of-equilibrium decay of the lighter messenger $\eta_{1}$. Subsequently, as long as the transmission between the produced left- and right-handed asymmetries is prevented from coming into thermal equilibrium at least until after the electroweak phase transition, the righthanded asymmetry could be hidden from the sphalerons and then the left-handed lepton number gets converted into the baryon asymmetry.

It is reliable to determine the final baryon asymmetry by exactly solving the full Boltzmann equations, which is beyond the scope of the present demonstration and will be presented elsewhere. For convenience, we will adopt the conventional approximate expression for the asymmetry of the right-handed neutrinos [8]:

$$
Y_{\nu_{R}} \equiv \frac{n_{\nu_{R}}}{s} \simeq \begin{cases}\varepsilon_{R 1} / g_{*}, & \text { for } K \ll 1, \\ 0.3 \varepsilon_{R 1} /\left[g_{*} K(\ln K)^{0.6}\right], & \text { for } K \gg 1,\end{cases}
$$

where $s$ is the entropy density and $g_{*}=\mathcal{O}(100)$ is the number of relativistic degrees of freedom in the early universe. The parameter $K$ stands for the ratio of the total decay width of $\eta_{1}$ to the expansion rate of the universe:

$$
\left.K \equiv \frac{\Gamma_{1}}{2 H(T)}\right|_{T=M_{\eta_{1}}}=\left(\frac{16 \pi^{3} g_{*}}{45}\right)^{-\frac{1}{2}} \frac{M_{\mathrm{Pl}} \Gamma_{1}}{M_{\eta_{1}}^{2}},
$$

where $H(T)=\left(8 \pi^{3} g_{*} / 90\right)^{1 / 2} T^{2} / M_{\mathrm{Pl}}$ is Hubble constant and $M_{\mathrm{Pl}} \simeq 1.22 \times 10^{19} \mathrm{GeV}$ is Planck mass.

It is clear from Eq. (12) that we must have at least two heavy messengers for the $\nu_{R}-\nu_{R}^{c}$ asymmetry to be nonzero, hence the neutrino mass matrix described in Eq. (3) should be expanded as,

$$
M_{\nu}=v u\left[\frac{y_{1} \rho_{1}}{M_{\eta_{1}}^{2}}+\frac{y_{2} \rho_{2}}{M_{\eta_{2}}^{2}}\right] .
$$

Under the limit of $M_{\eta_{1}} \ll M_{\eta_{2}}$ and for $\rho_{i} \lesssim M_{\eta_{i}}$, we can neglect the contribution from $\eta_{2}$ in the above equation. Thus, we further derive the neutrino masses as,

$$
\begin{aligned}
\sum_{j=1}^{3} m_{j}^{2} & =\operatorname{Tr}\left(M_{\nu}^{\dagger} M_{\nu}\right) \simeq \operatorname{Tr}\left(y_{1}^{\dagger} y_{1}\right) \frac{\rho_{1}^{2} v^{2} u^{2}}{M_{\eta_{1}}^{4}} \\
& =\frac{(4 \pi)^{5} g_{*}}{45} B_{f} B_{s} K^{2} \frac{v^{2} u^{2}}{M_{\mathrm{Pl}}^{2}},
\end{aligned}
$$

where $m_{j}(j=1,2,3)$ are the neutrinos masseigenvalues. $B_{f}$ and $B_{s}$ are the branching ratios of $\eta_{1}$ decaying to the fermions and the scalars, respectively,

$$
\begin{aligned}
& B_{f} \equiv \frac{\sum_{\alpha \beta} \Gamma\left(\eta_{1} \rightarrow \psi_{L \alpha} \nu_{R \beta}^{c}\right)}{\Gamma_{1}}=\frac{\operatorname{Tr}\left(y_{1}^{\dagger} y_{1}\right) M_{\eta_{1}}}{8 \pi \Gamma_{1}}, \\
& B_{s} \equiv \frac{\Gamma\left(\eta_{1} \rightarrow \phi \chi\right)}{\Gamma_{1}}=\frac{\rho_{1}^{2} / M_{\eta_{1}}}{8 \pi \Gamma_{1}}
\end{aligned}
$$


We note that $B_{f}$ and $B_{s}$ obey the relationship,

$$
B_{f}+B_{s}=1, \quad \Longrightarrow \quad B_{f} B_{s} \leqslant \frac{1}{4} .
$$

It is convenient to express $K$ as a function of the neutrino masses,

$$
K=\left[\frac{(4 \pi)^{5} g_{*}}{45}\right]^{-\frac{1}{2}}\left(B_{f} B_{s}\right)^{-\frac{1}{2}} \frac{\bar{m} M_{\mathrm{Pl}}}{v u}
$$

where the quadratic mean $\bar{m}$ of the neutrino masses is defined by $\bar{m}^{2} \equiv \sum_{j=1}^{3} m_{j}^{2}$.

For illustration below, let us choose,

$$
r \equiv \frac{M_{\eta_{1}}}{M_{\eta_{2}}}=\frac{\rho_{1}}{\rho_{2}}, \quad y_{2}=y_{1} e^{i \delta},
$$

where $\delta$ denotes the relative phase between $y_{1}$ and $y_{2}$. So, we can derive the CP-asymmetry from Eq. (12),

$$
\begin{aligned}
\varepsilon_{R 1} & =\frac{1}{4 \pi} \frac{\operatorname{Tr}\left(y_{1}^{\dagger} y_{1}\right) \rho_{1}^{2}}{\operatorname{Tr}\left(y_{1}^{\dagger} y_{1}\right) M_{\eta_{1}}^{2}+\rho_{1}^{2}} r \sin \delta \\
& =\left[\frac{(4 \pi)^{3} g_{*}}{45}\right]^{\frac{1}{2}} B_{f} B_{s} K \frac{M_{\eta_{1}}}{M_{\mathrm{Pl}}} r \sin \delta \\
& =\frac{\left(B_{f} B_{s}\right)^{\frac{1}{2}}}{4 \pi} \frac{M_{\eta_{1}} \bar{m}}{v u} r \sin \delta .
\end{aligned}
$$

For the inputs of $B_{f} B_{s}=1 / 4, \sin \delta=-1, \quad r=0.1$, $u=1 \mathrm{TeV}, \quad M_{\eta_{1}}=1.15 \times 10^{12} \mathrm{GeV}$ and $\bar{m}=0.1 \mathrm{eV}$, we derive the sample predictions, $\varepsilon_{R 1} \simeq-2.6 \times 10^{-6}$ and $K \simeq 16$. In consequence, applying Eqs. (5) and (13), we arrive at, $n_{B} / s \simeq 8.7 \times 10^{-11}$. From this we can deduce, $n_{B} / n_{\gamma} \simeq 7.04 n_{B} / s \simeq 6.1 \times 10^{-10}$, which agrees well to the observation [9], $n_{B} / n_{\gamma}=(6.0965 \pm 0.2055) \times 10^{-10}$, where $n_{\gamma}$ is the current photon number density.

In conclusion, we have presented a new Dirac Seesaw scenario to simultaneously explain the origin of neutrino masses and the generation of baryon asymmetry in the universe. This is achieved by extending the visible content of the SM with a hidden sector which has new $U(1)_{X}$ gauge symmetry and is composed of three right-handed singlet neutrinos and one singlet Higgs. The messages between these two sectors are mediated by the heavy messenger scalar doublets. The heavy messengers can help to highly suppress the neutrino masses and also decay efficiently to provide the baryon asymmetry. Further phenomenological consequences from the hidden sector (including the new gauge boson $Z^{\prime}$ from the broken $U(1)_{X}$ [10]) are interesting and will be studied elsewhere.

\section{APPENDIX A}

The general scalar potential in our model is

$$
\begin{aligned}
& V\left(\phi, \chi, \eta_{i}\right) \\
&=-\mu^{2} \phi^{\dagger} \phi+\lambda\left(\phi^{\dagger} \phi\right)^{2}-\sigma^{2} \chi^{\dagger} \chi+\kappa\left(\chi^{\dagger} \chi\right)^{2}+\xi \phi^{\dagger} \phi \chi^{\dagger} \chi \\
&+\sum_{i j k \ell}\left\{M_{\eta_{i}}^{2} \eta_{i}^{\dagger} \eta_{i}+\delta_{i j k \ell} \eta_{i}^{\dagger} \eta_{j} \eta_{k}^{\dagger} \eta_{\ell}\right. \\
&+\alpha_{i j} \phi^{\dagger} \phi \eta_{i}^{\dagger} \eta_{j}+\beta_{i j} \phi^{\dagger} \eta_{i} \eta_{j}^{\dagger} \phi+\gamma_{i j} \chi^{\dagger} \chi \eta_{i}^{\dagger} \eta_{j} \\
&\left.-\left[\rho_{i} \chi \eta_{i}^{\dagger} \phi+\text { h.c. }\right]\right\},
\end{aligned}
$$

where the indices $(i, j, k, \ell)=1,2, \cdots$, denote the number of the messenger doublets. Without losing generality, the tree-level mass matrix for the scalar doublets $\eta_{i}$ have been chosen to be diagonal and real. We then analyze the potential as a function of the scalar VEVs, $v \equiv\langle\phi\rangle$, $u \equiv\langle\chi\rangle$ and $w_{i} \equiv\left\langle\eta_{i}\right\rangle$,

$$
\begin{aligned}
V\left(v, u, w_{i}\right) & \\
= & -\mu^{2} v^{2}+\lambda v^{4}-\sigma^{2} u^{2}+\kappa u^{4}+\xi v^{2} u^{2} \\
& +\sum_{i j k \ell}\left\{M_{\eta_{i}}^{2} w_{i}^{2}+\delta_{i j k l} w_{i} w_{j} w_{k} w_{\ell}\right. \\
& \left.+\left(\alpha_{i j}+\beta_{i j}\right) v^{2} w_{i} w_{j}+\gamma_{i j} u^{2} w_{i} w_{j}-2 \rho_{i} v u w_{i}\right\} .
\end{aligned}
$$

Taking the extreme conditions, $0=\partial V / \partial v=\partial V / \partial u=$ $\partial V / \partial w_{i}$, we obtain

$$
\begin{aligned}
0= & -2 \mu^{2} v+4 \lambda v^{3}+2 \xi v u^{2} \\
& +\sum_{i j}\left[2\left(\alpha_{i j}+\beta_{i j}\right) v w_{i} w_{j}-2 \rho_{i} u w_{i}\right], \\
0= & -2 \sigma^{2} u+4 \kappa u^{3}+2 \xi v^{2} u \\
& +\sum_{i j}\left[2 \gamma_{i j} u w_{i} w_{j}-2 \rho_{i} v w_{i}\right], \\
0= & \sum_{j k \ell}\left[2 M_{\eta_{i}}^{2} w_{i}+4 \delta_{i j k \ell} w_{j} w_{k} w_{\ell}\right. \\
& \left.+2\left(\alpha_{i j}+\beta_{i j}\right) v^{2} w_{j}+2 \gamma_{i j} u^{2} w_{j}-2 \rho_{i} v u\right] .
\end{aligned}
$$

As before, we will choose $M_{\eta_{i}} \gg v, u$ and $\rho_{i} \lesssim M_{\eta_{i}}$, and thus we deduce $w_{i}$ [11] from solving Eq. (A5),

$$
w_{i} \simeq \frac{\rho_{i} v u}{M_{\eta_{i}}^{2}} \ll v, u .
$$

Subsequently, we have

$$
\begin{aligned}
& v^{2}=\frac{2 \kappa \mu^{2}-c \sigma^{2}}{4 \lambda \kappa-c^{2}}, \\
& u^{2}=\frac{2 \lambda \sigma^{2}-c \mu^{2}}{4 \kappa \lambda-c^{2}},
\end{aligned}
$$

where the coefficient $c \equiv \xi-\sum_{i} \rho_{i}^{2} / M_{\eta_{i}}^{2}$. In order to ensure $v$ and $u$ to be nonzero, we can choose the parameters 
$\mu^{2}, \sigma^{2}, \lambda, \kappa$ and $c$ to satisfy the following conditions,

$$
\left\{\begin{array}{l}
c \neq 0, \\
4 \kappa \lambda-c^{2}>0, \\
\frac{c}{2 \kappa}<\frac{\mu^{2}}{\sigma^{2}}<\frac{2 \lambda}{c},
\end{array} \text { or, } \quad c=0 .\right.
$$

[1] A different and inspiring approach to the hidden sector was recently suggested by B. Patt and F. Wilczek, hep-ph/0605188.

[2] P. Minkowski, Phys. Lett. B 67, 421 (1977); T. Yanagida, in Proceedings of the Workshop on Unified Theory and the Baryon Number of the Universe, edited by O. Sawada and A. Sugamoto (KEK, Tsukuba, 1979), p. 95; M. Gell-Mann, P. Ramond, and R. Slansky, in Supergravity, edited by F. van Nieuwenhuizen and D. Freedman (North Holland, Amsterdam, 1979), p. 315; S.L. Glashow, in Quarks and Leptons, edited by M. Lévy et al. (Plenum, New York, 1980), p. 707; R.N. Mohapatra and G. Senjanović, Phys. Rev. Lett. 44, 912 (1980).

[3] S. Weinberg, Phys. Rev. Lett. 43, 1566 (1979).

[4] K. Dick, M. Lindner, M. Ratz, and D. Wright, Phys. Rev. Lett. 84, 4039 (2000); H. Murayama and A. Pierce, Phys. Rev. Lett. 89, 271601 (2002); B. Thomas and M. Toharia, Phys. Rev. D 73, 063512 (2006); D.G. Cerdeno, A. Dedes, and T.E.J. Underwood, JHEP 0609, 067 (2006); B. Thomas and M. Toharia, hep-ph/0607285

[5] V.A. Kuzmin, V.A. Rubakov and M.E. Shaposhnikov, Phys. Lett. B 155, 36 (1985); R.N. Mohapatra and X. Zhang, Phys. Rev. D 45, 2699 (1992).
[6] M. Fukugita and T. Yanagida, Phys. Lett. B 174, 45 (1986); P. Langacker, R.D. Peccei, and T. Yanagida, Mod. Phys. Lett. A 1, 541 (1986); M.A. Luty, Phys. Rev. D 45, 455 (1992); R.N. Mohapatra and X. Zhang, Phys. Rev. D 46, 5331 (1992). For a recent review, W. Buchmuller, R.D. Peccei, and T. Yanagida, Ann. Rev. Nucl. Part. Sci. 55, 311 (2005).

[7] Dirac neutrinos are also suitable in the mechanism of baryogenesis via neutrino oscillations, E.Kh. Akhmedov, V.A. Rubakov, and A.Yu. Smirnov, Phys. Rev. Lett. 81, 1359 (1998).

[8] E.W. Kolb and M.S. Turner, The Early Universe, Addison-Wesley, Reading, MA, 1990; H.B. Nielsen and Y. Takanishi, Phys. Lett. B 507, 241 (2001).

[9] D.N. Spergel et al., astro-ph/0603449.

[10] For a recent review, T.G. Rizzo, hep-ph/0610104.

[11] Our mechanism of generating small VEVs for heavy scalar doublets is similar to the type-II seesaw model where heavy scalar triplets get small VEVs for the mass generation of Majorana neutrinos, E. Ma and U. Sarkar, Phys. Rev. Lett. 80, 5716 (1998). 